\title{
Nanovoid Cavitation by Dislocation Emission in Aluminum
}

\author{
Jaime Marian, Jaroslaw Knap, and Michael Ortiz \\ Division of Engineering and Applied Science, California Institute of Technology, Pasadena, California 91125, USA
}

(Received 22 March 2004; published 14 October 2004)

\begin{abstract}
This Letter is concerned with the determination of the transition paths attendant to nanovoid growth in aluminum under hydrostatic tension. The analysis is, therefore, based on energy minimization at $0 \mathrm{~K}$. Aluminum is modeled by the Ercolessi-Adams embedded-atom method, and spurious boundary artifacts are mitigated by the use of the quasicontinuum method. Our analysis reveals several stages of pressure buildup separated by yield points. The first yield point corresponds to the formation of highly stable tetrahedral dislocation junctions around the surfaces of the void. The second yield point is caused by the dissolution of the tetrahedral structures and the emission of conventional $\frac{1}{2}\langle 110\rangle\{111\}$ and anomalous $\frac{1}{2}\langle 110\rangle\{001\}$ dislocation loops.
\end{abstract}

DOI: 10.1103/PhysRevLett.93.165503

PACS numbers: 61.72.-y, 02.70.-c, 46.15.-x

Spallation damage under dynamic tensile loading is characterized by the emergence of distributed microcracks or voids in a narrow region of the material, or spall plane, which may result in the catastrophic failure of the specimen. Spall has been widely studied experimentally by means of gas-gun driven plate-impact tests (e.g., $[1,2])$, high-intensity, pulsed laser shock generators [3-5], and other experimental techniques (cf. [6] and references therein). Observations, however, are for the most part restricted to free-surface velocity measurements and post mortem examination of the specimens and, therefore, are necessarily indirect.

Molecular dynamics (MD) suggests itself as a natural approach for understanding the intrinsic mechanisms underlying the evolution of nanosized voids [7-9]. These studies reveal, among other useful insights, that nanovoids exceeding a pressure and temperaturedependent critical size grow by the emission of dislocations and by coalescence with neighboring voids. However, MD is particularly well suited to the study of void growth at very high strain rates, often greatly in excess of those attained experimentally. In addition, reliance on periodic boundary conditions limits the range of elapsed times which can be examined by MD and may introduce undesirable artifacts. Continuum estimates [10] suggest that dynamic effects are indeed negligible for small voids at moderate-to-high strain rates. This points to the need to complement MD studies with a detailed analysis of the equilibrium energy landscape and transition paths accessible to expanding nanovoids.

The technique that we use in order to carry out such an analysis is the quasicontinuum (QC) method. QC is a method for systematically coarse-graining lattice statics models. The method starts with the complete atomistic system and appends kinematic constraints which restrict the configuration space of the crystal. The kinematic constraints are based on the selection of representative atoms and the use of finite-element interpolation. In order to avoid full lattice sums, cluster summation rules are also used. By virtue of these rules, only atoms in small clusters surrounding the representative atoms need to be visited in computing the effective out-of-balance forces. Finally, the selection of representative atoms is performed adaptively based on local measures of deformation and longest-edge tetrahedral bisection. The adaption tolerances are set such that full atomistic resolution is guaranteed in the presence of dislocations. The quasicontinuum method uses empirical potentials directly as the sole description of material behavior and contains fully atomistic lattice statics and continuum elasticity as special limits. Details of the implementation and an analysis of the accuracy and convergence of the method may be found in [11]. Here, the computational cell concerns a $432 a_{0} \times$ $432 a_{0} \times 432 a_{0}$ cube $174 \mathrm{~nm}$ in size of fcc ErcolessiAdams [12] Al oriented along cubic lattice directions and nominally containing $3.2 \times 10^{8}$ atoms. An equiaxed $5-\mathrm{nm}$ void is initially created in the center of the cell with full atomistic resolution being provided $a b$ initio within a $16 a_{0} \times 16 a_{0} \times 16 a_{0}$ region surrounding the void. The triangulation is rapidly coarsened with distance to the void elsewhere, resulting in an initial computational mesh containing 31933 nodes.

Recent experimental data [13] suggest that the material response within a strong shock is essentially volumetric. We therefore drive the void expansion by prescribing pure dilatational displacements over the exterior boundary of the computational cell. Thus, if $\epsilon$ is the nominal axial strain imparted on the sample, we increase $\epsilon$ steadily from $\epsilon=0$ to $\epsilon=10.5 \%$ by $0.1 \%$ increments. At each loading step, a new stable equilibrium configuration is obtained by using the Polak-Ribiere variant of the conjugate gradient algorithm [14]. The computational mesh is then adapted so as to ensure full atomistic resolution locally well in advance of the passage of dislocations. In order to reliably identify the defects in the crystal we employ the centrosymmetry deviation parameter [15]. For a partial dislocation core atom in Al the deviation parameter is $2.1 \AA^{2}$, between 5 and $20 \AA^{2}$ for a stacking fault, and $24.4 \AA^{2}$ for a $\{111\}$ free surface. In all subsequent dislocation structure plots, atoms are colored ac- 
cording to the relative magnitude of their centrosymmetry deviation parameter with blue and red corresponding to free surfaces and partial dislocation cores, respectively, while atoms belonging to stacking faults are identified by means of a green-to-orange color gradation.

The computed pressure vs volumetric strain $\left(p-\varepsilon_{v}\right)$ and normalized void growth $\left(\Delta V_{v}-\varepsilon_{v}\right)$ curves are plotted in Fig. 1. The pressure $p$ is calculated from the external tractions acting on the boundary of the computational domain. We define the volumetric strain as $\varepsilon_{v}=$ $(1+\epsilon)^{3}-1$. Finally, $\Delta V_{v} / V_{v 0}$ denotes the void volume increment with respect to the initial void volume of $\sim 72.9 \mathrm{~nm}^{3}$.

Three main stages of rapid pressure buildup punctuated by inflection or yield points are clearly discernible in the $\left(p-\varepsilon_{v}\right)$ curve in Fig. 1: (i) an initial elastic stage up to the first yield point at $\varepsilon_{v}=17.2 \%$, characterized by elastic expansion of the void without dislocation emission; (ii) a second stage of hardening up to a second yield point at $\varepsilon_{v}=30.8 \%$, characterized by the formation of highly stable tetrahedral dislocation structures around the surface of the void; and (iii) a third stage of hardening characterized by the emission of a burst of dislocations from the void. Dislocation emission episodes result in sharp growth spurts of the void. However, these local instabilities are confined by the surrounding elastic matrix and, in particular, the average pressure in the cell exhibits no drops. We refer to the various stages of deformation as stages I, II, and III, respectively, for ease of reference. We proceed to describe the deformation mechanisms underlying these stages.

The first dislocation structures emerge immediately following the first yield point at a pressure of $19.2 \mathrm{GPa}$,

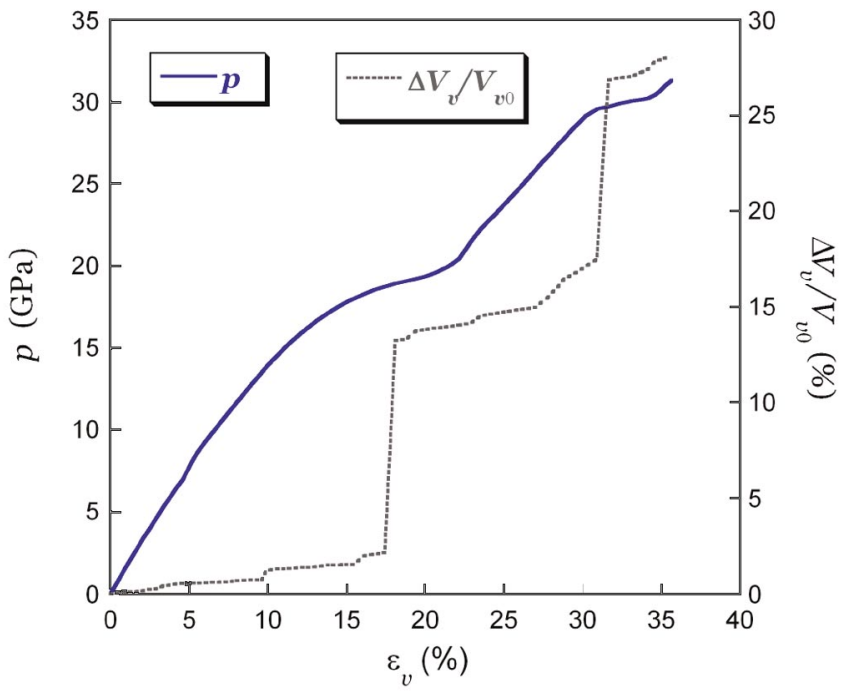

FIG. 1 (color). Computed hydrostatic pressure and normalized void volume expansion $\Delta V_{v} / V_{v 0}$ vs volumetric deformation $\varepsilon_{v}$ of the sample. Three main regimes, separated by two yield points, can be distinguished in the curve, namely, an elastic regime, lock hardening, and work hardening. which thus may be regarded as the critical pressure for the inception of plastic cavitation. The computed cavitation pressure of $19.2 \mathrm{GPa}$ is in good agreement with experimentally measured values [3], results of first-principles calculations [16], and Grady's [17] formula for the theoretical spall strength, which gives a value of $18.2 \mathrm{GPa}$.

The dislocation structures that form immediately after the first yield point are shown in Fig. 2. A set of tetrahedral dislocation junctions symmetrically distributed on all six $\langle 100\rangle$ apices of the void can be clearly discerned in the figure. A detailed analysis of the dislocation structures reveals sets of four tetrahedra converging at a single point. The tetrahedra are composed of $\{111\}$ stackingfault surfaces bounded, alternatively, by $\frac{1}{6}\langle 110\rangle$ stair-rodtype sessile dislocations and $\frac{1}{2}\langle 110\rangle$ perfect dislocations. These fourfold assemblies of stacking-fault tetrahedra are seen to form exceptionally strong junctions, similar to the threefold-symmetric dislocation structures found previously in atomistic simulations of Al nanoindentation [18]. Indeed, the formation of extended stacking-fault nodes is a well-documented phenomenon in fcc metals (cf. [19]).

The stair-rod dislocation structures can be attributed to the Lomer-Cottrell reaction, in which two perfect dislocations split into partials react along the intersection line

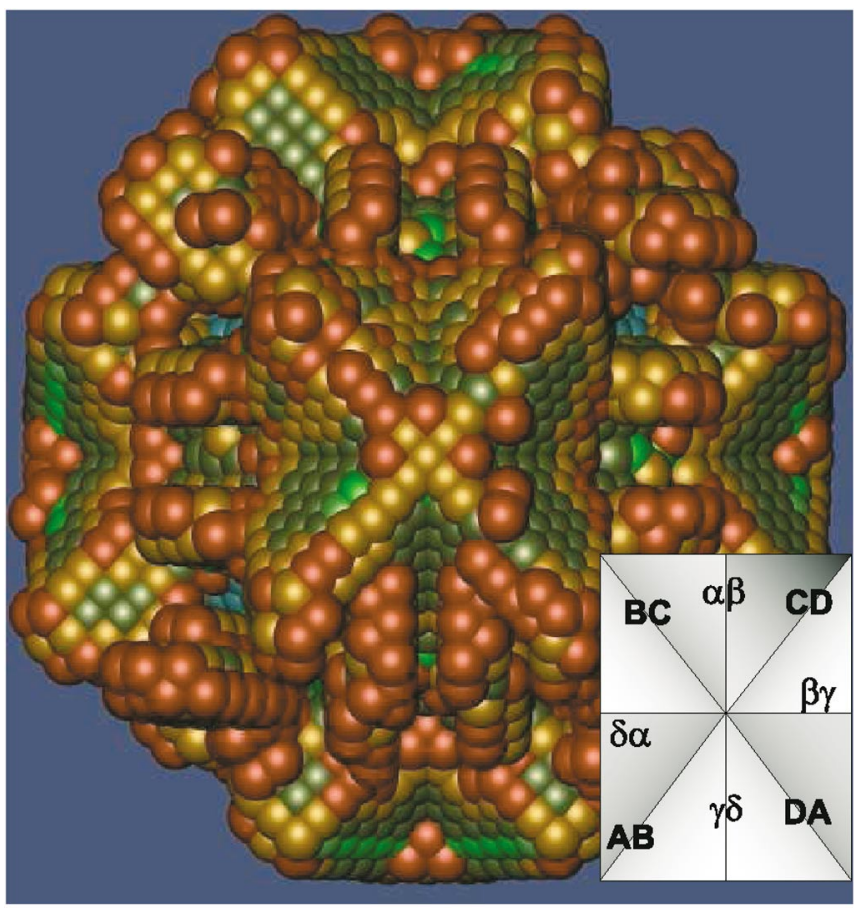

FIG. 2 (color). [010] view of the dislocation structures generated at the first yield point. The four tetrahedra have one common vertex at which the sum of Burgers vectors of the converging stair rod and perfect dislocations is conserved. A schematic diagram showing the four tetrahedra and the Burgers vectors (in Thompson's notation) corresponding to all dislocation junctions is provided in the inset. 
of their respective $\{111\}$ glide planes to form a sessile junction. More specifically,

$$
\begin{gathered}
\frac{1}{2}[101]+\frac{1}{2}[01 \overline{1}] \rightarrow \frac{1}{6}[112]+\frac{1}{6}[11 \overline{2}]+\frac{1}{6}[110], \\
D A+A C \rightarrow D \gamma+\delta C+\gamma \delta,
\end{gathered}
$$

where the first two product dislocations are Shockley partials and the last one is the stair rod. The stair-rod dislocation exerts a repulsive force on the two remaining partials. These, in turn, recombine with the two repulsed partials of the adjacent tetrahedra to form perfect dislocations. These so-called Lomer-Cottrell junctions act as strong barriers to further glide on the two $\{111\}$ planes involved and remain locked for as long as the two trailing partials of the converging dislocations remain on separate planes.

The formation of highly stable tetrahedral locks temporarily inhibits further dislocation activity and the initial yield point is followed by a second stage of rapid pressure buildup (stage II). During this lock-hardening stage, the $\left(p-\varepsilon_{v}\right)$ curve grows monotonically. Eventually, at a pressure of approximately $29 \mathrm{GPa}$ the tetrahedral structures lose stability and a burst of dislocation emission takes place. The emitted dislocations rapidly expand into the surrounding matrix, resulting in a second void growth spurt of $\sim 9 \%$. The observed dislocation distribution is qualitatively similar to that predicted by Lubarda et al. in their analytical study of void growth [5].

The swift and unrestricted expansion of the glissile loops requires that the atomistic region surrounding the void grow accordingly in order to avoid numerical artifacts. This growth is illustrated in Fig. 3, where the QC mesh at $30.8 \%$ volumetric strain is shown. The corresponding dislocation structures are shown in Fig. 4.

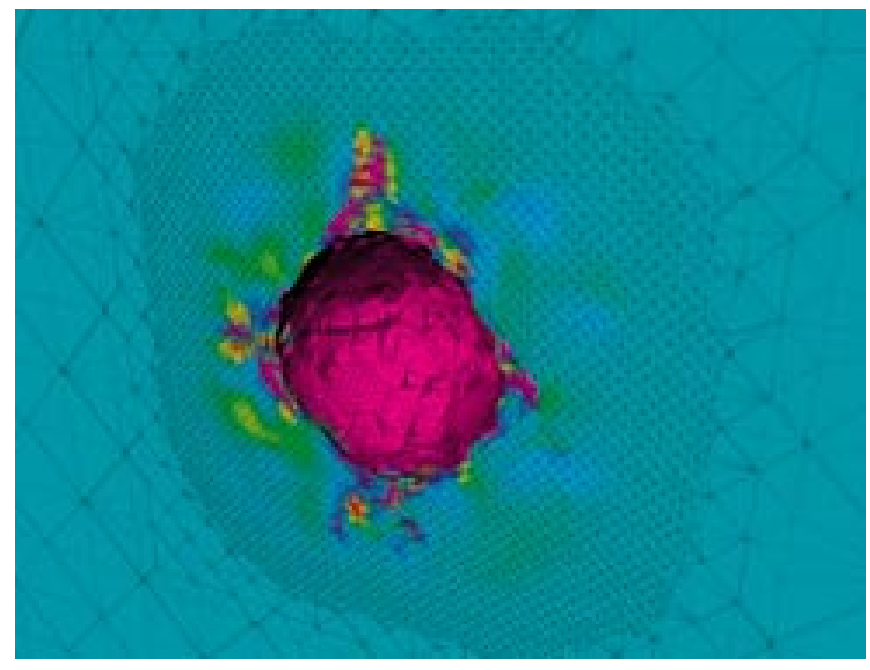

FIG. 3 (color). View of the QC computational mesh (cut through $x=0)$ shortly after the second yield point $\left(\varepsilon_{v}=\right.$ $30.8 \%$ ). The figure is color-coded according to the magnitude of the displacements perpendicular to the plane of the cut.
Interestingly, in addition to the $\frac{1}{2}\langle 110\rangle\{111\}$-type dislocations expected in fcc metals, large, petal-like shear loops of $\frac{1}{2}\langle 110\rangle\{001\}$ character can be clearly observed in the figure. Slip on $\{100\}$ planes, although uncommon in closepacked metals, has been reported for $\mathrm{Al}[20,21]$ and postulated theoretically by Cottrell and others $[22,23]$. Thus, the reaction

$$
\begin{gathered}
\frac{1}{6}[112]+\frac{1}{6}[11 \overline{2}]+\frac{1}{6}[110] \rightarrow \frac{1}{2}[110], \\
D \gamma+\delta C+\gamma \delta \rightarrow D C
\end{gathered}
$$

is common in metals with high stacking-fault energy such as Al. The two trailing partials of Eq. (1) can further collapse upon the stair-rod dislocation and give rise to a perfect $\frac{1}{2}[110]$ dislocation capable of gliding on (001)-type planes. The classical explanation for this behavior (cf. [20]) is based on the assumption that the split Lomer-Cottrell dislocation has to recombine over a certain segment length and form a total dislocation which bows out on a $\{100\}$ plane, giving rise to growing loops. Because of the repulsive nature of the three reactant dislocations in reaction (3), this can be achieved only by increasing the level of applied stress, which is reflected in the $\left(p-\varepsilon_{v}\right)$ evolution corresponding to stage II. Notably, the $\frac{1}{2}\langle 110\rangle$ dislocation glide on $\{100\}$ planes has also been observed at moderate stresses in materials with stackingfault energies well below that of $\mathrm{Al}$ [20]. Our analysis of the dislocation structures further suggests that during the

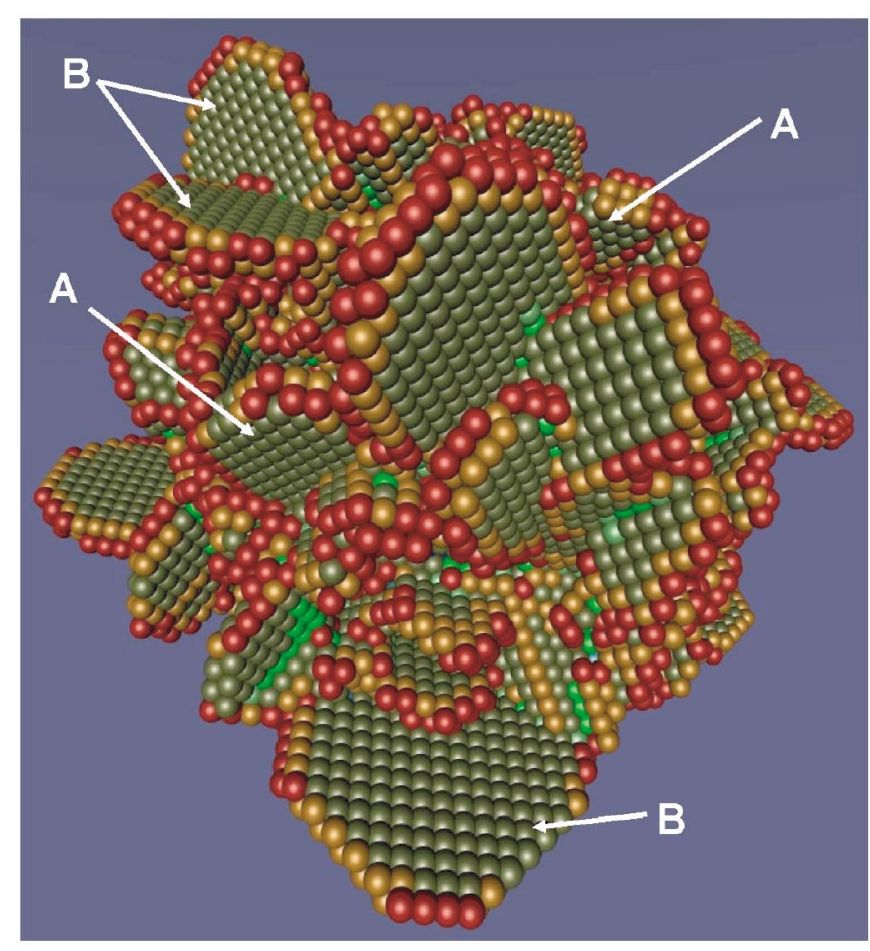

FIG. 4 (color). Dislocation structures at $\varepsilon_{v}=30.8 \%$. The activated slip systems include the conventional $\frac{1}{2}\langle 110\rangle\{111\}$ (type A) and the anomalous $\frac{1}{2}\langle 110\rangle\{001\}$ (type B). 


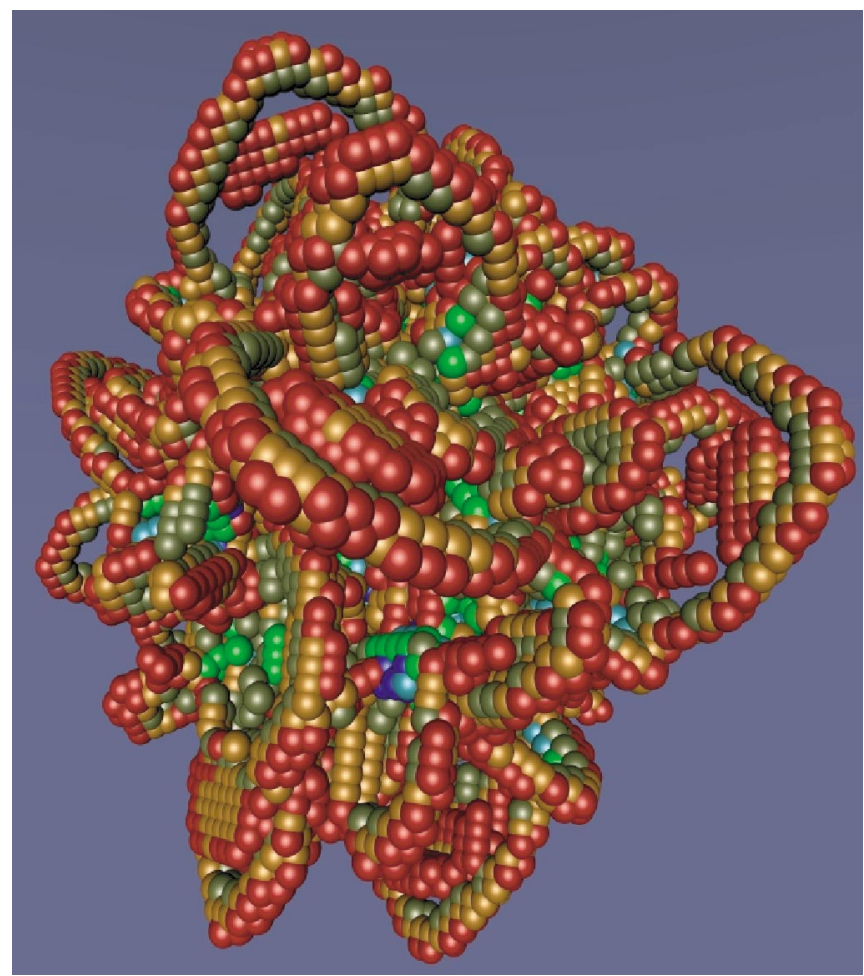

FIG. 5 (color). Final detachment of the $\frac{1}{2}\langle 110\rangle\{001\}$ dislocations corresponding to a dilatation of $34.5 \%$.

breakdown of the Lomer-Cottrell locks the locking points remain fixed, effectively pinning the loops. The dislocation loops initially grow without detaching from the void, and they leave a shear plate behind. We attribute the temporary presence of this unstable shear plate to the very high stresses present inside the sample.

Upon increased loading, the dislocation ensemble grows steadily and the size of the void increases. The shear stress in our sample decreases rapidly away from the void surface, as $\sim 1 / r^{2}$. This explains why the loops reach their maximum size at a distance of about 5-7 nm from the void, which is when the shear stress falls below the critical glide stress on $\{001\}$ planes. The hardening observed in the $\left(p-\varepsilon_{v}\right)$ curve is lost at pressures of about $29 \mathrm{GPa}$, and $p$ can be seen to soften progressively up to $\varepsilon_{v}=34.5 \%$. The dislocation activity around the void becomes exceedingly complex due to the juxtaposition of existing structures and the nucleation of new ones. The $\frac{1}{2}\{110\}$ shear loops are eventually observed to detach and the extended fault plates to dissolve (cf. Fig. 5). Following this stage, the growth of the loops slows down again and a new hardening stage sets in.

In conclusion, we have studied the transition paths attendant to nanovoid growth in Al. Our analysis reveals several stages of pressure buildup separated by yield points. The first yield point corresponds to the formation of highly stable tetrahedral dislocation junctions around the boundary of the void. The tetrahedral structures are bounded by stacking-fault surfaces, stair-rod dislocations, and perfect dislocations. The second yield point is caused by the dissolution of the tetrahedral structures and the emission of conventional $\left(\frac{1}{2}\langle 110\rangle\{111\}\right)$ and anomalous $\left(\frac{1}{2}\langle 110\rangle\{001\}\right)$ dislocation loops. To the best of knowledge, these deformation mechanisms had not previously been observed in atomistic simulations of expanding voids. Our analysis suggests that the initial stages of expansion of a nanovoid in $\mathrm{Al}$ under pressure are the result of a number of highly complex transitions that negotiate an exceedingly rugged energy landscape.

Support from the DOE through Caltech's ASCI/ASAP Center for the Simulation of the Dynamic Response of Solids is gratefully acknowledged.

[1] Q. Johnson, A. Mitchell, R. Norris-Keeler, and L. Evans, Phys. Rev. Lett. 25, 1099 (1970).

[2] S. Nemat-Nasser, T. Okinaka, V. Nesterenko, and M. Liu, Philos. Mag. A 78, 1151 (1998).

[3] E. Moshe et al., J. Appl. Phys. 83, 4004 (1998).

[4] D. Kalantar et al., Phys. Plasmas 10, 1569 (2003).

[5] V. Lubarda, M. Schneider, D. Kalantar, B. Remington, and M. Meyers, Acta Mater. 52, 1397 (2004).

[6] D. E. Grady and M.E. Kipp, in High-Pressure Shock Compression of Solids, edited by J. R. Asay and M. Shahinpoor (Springer-Verlag, New York, 1993), pp. 265-322.

[7] J. Belak, J. Comput,-Aided Mater. Des. 5, 193 (1998).

[8] A. Strachan, T. Cagin, and W. Goddard, Phys. Rev. B 63, 060103 (2001).

[9] R. Rudd and J. Belak, Comput. Mater. Sci. 24, 148 (2002).

[10] M. Ortiz and A. Molinari, J. Appl. Mech. 59, 48 (1992).

[11] J. Knap and M. Ortiz, J. Mech. Phys. Solids 49, 1899 (2001).

[12] F. Ercolessi and J. Adams, Europhys. Lett. 26, 583 (1994).

[13] A. Loveridge-Smith et al., Phys. Rev. Lett. 86, 2349 (2001).

[14] W. Press, W. Vetterling, S. Teutoslky, and B. Flannery, Numerical Recipes in $\mathrm{C}++$ (Cambridge University Press, Cambridge, 2002), 2nd ed.

[15] C. Kelchner, S. Plimpton, and J. Hamilton, Phys. Rev. B 58, 11085 (1998).

[16] D. Clatterbuck, C. Krenn, M. Cohen, and J. Morris, Phys. Rev. Lett. 91, 135501 (2003).

[17] D. Grady, J. Mech. Phys. Solids 3, 353 (1988).

[18] K. Van Vliet, J. Li, T. Zhu, S. Yip, and S. Suresh, Phys. Rev. B 67, 104105 (2003).

[19] D. Hull and D. Bacon, Introduction to Dislocations (Pergamon Press, Oxford, 1984), 3rd ed., p. 158.

[20] H. Karnthaler, Philos. Mag. 38, 141 (1978).

[21] A. Korner and H. Karnthaler, Philos. Mag. 42, 753 (1980).

[22] A. Cottrell, Philos. Mag. 43, 645 (1952).

[23] J. De Hosson, O. Kanert, and A. Sleeswyk, Dislocations in Solids, edited by F. R. N. Nabarro (North-Holland Publishing Company, New York, 1983), Chap. 32, p. 452. 